Revue

Revue de l'histoire des religions

de Ihistoire des religions

$4 \mid 2012$

Varia

\title{
Les Sirènes : du chant mortel à la musique des sphères. Lectures homériques et interprétations platoniciennes
}

The Sirens: from the deadly song to the music of the spheres. Homeric readings and Platonic interpretations

\section{Carine Van Liefferinge}

\section{OpenEdition}

\section{Journals}

Édition électronique

URL : http://journals.openedition.org/rhr/7980

DOI : $10.4000 /$ rhr.7980

ISSN : 2105-2573

\section{Éditeur}

Armand Colin

\section{Édition imprimée}

Date de publication : 1 décembre 2012

Pagination : 479-501

ISBN : 978-2200-92796-7

ISSN : 0035-1423

\section{Référence électronique}

Carine Van Liefferinge, « Les Sirènes : du chant mortel à la musique des sphères. Lectures homériques et interprétations platoniciennes », Revue de l'histoire des religions [En ligne], 4 | 2012, mis en ligne le 01 décembre 2015, consulté le 10 décembre 2020. URL : http://journals.openedition.org/rhr/7980 ; DOI : https://doi.org/10.4000/rhr.7980 


\section{Les Sirènes : du chant mortel à la musique des sphères Lectures homériques et interprétations platoniciennes}

Par leur nature et le lieu qu'elles occupent, les Sirènes odysséennes sont marquées par l'ambiguïté. C'est précisément celle-ci qui leur accorda une place dans le discours des philosophes, particulièrement chez Platon. Dans le mythe d'Er de la République, il se sert de leur caractère essentiel pour insérer un curieux excursus astronomique dans une théorie eschatologique. Cette ambiguïté est définitivement levée par Proclus. Surinterprétant le discours de Platon, il y décèle trois espèces de Sirènes, les célestes, les terrestres et les souterraines. Il assure ainsi la cohérence du texte platonicien, le concilie avec le mythe et l'éclaire à la lumière du rituel théurgique, dans une démarche inaugurée par Jamblique.

The Sirens: from the deadly song to the music of the spheres. Homeric readings and Platonic interpretations

By their nature and the place which they occupy, the Homeric Sirens appear as ambiguous beings. It is precisely this characteristic which assigned them a place in philosophical discourse, particularly in Plato's. In the myth of Er in the Republic, he uses their ambiguous nature to insert a strange astronomical excursus into an eschatological theory. This ambiguity will be definitively removed by Proclus. He overinterprets the Platonic text, which, according to him, reveals three kinds of Sirens, celestial, terrestrial and subterranean. In this way, he finds cohesion within the Platonic text, reconciling it with the myth, and shedding light on it by means of the theurgical ritual as did Iamblichus before him. 


\section{LES SiRÈnES OdySSÉENNES, FIGURES AMBIGUËS À LA CROISÉE DES CHEMINS}

Le premier conseil que Circé donna à Ulysse lorsque le moment fut arrivé pour lui de reprendre la route était de fuir les Sirènes, leur voix ensorcelante et leur prairie en fleurs ${ }^{1}$. De ces figures mythiques, Homère ne précise ni l'origine, ni la forme ${ }^{2}$ : tout au plus apprenonsnous de l'usage du duel qu'elles sont deux - et encore n'est-ce pas une certitude ${ }^{3}$-, qu'elles séjournent sur une île qui se présente comme un pré au rivage blanchi par les ossements des malheureux que leur chant a envoûtés ${ }^{4}$, et qu'elles promettent à ceux qu'elles attirent par leur chant de repartir plus savants ${ }^{5}$ car elles savent, chantent-elles à Ulysse, tout ce qui s'est passé à Troie, ainsi que «tout ce qui se passe sur la terre nourricière $»^{6}$. Omniscientes sont donc les Sirènes, et prophétiques. En effet, elles sont qualifiées de l'adjectif $\theta \varepsilon \sigma \pi \varepsilon ́ \sigma 10 \varsigma^{7}$, qui renvoie étymologiquement à l'inspiration divine, et l'allusion du poète à leur voix «douce comme le miel $»^{8}$ suggère un rapprochement entre les Sirènes et les abeilles, animaux chargés d'un symbolisme poétique et prophétique?

1. Odyssée XII, 158-159.

2. Sur ce débat (étaient-elles femmes ou femmes-oiseaux ?) qui n'a pas sa place ici, voir notamment Gerald K. Gresseth, «The Homeric Sirens», dans Transactions and Proceedings of the American Philological Association 101, 1970, p. 203-218.

3. Homère parle des Sirènes au pluriel et, moins souvent, au duel. De cet usage, Eustathe attribue néanmoins à Homère d'avoir parlé de deux Sirènes anonymes (Commentarii in Dionysii periegetae orbis descriptionem 358, dans Geographi Graeci Minores, éd. C. Müller, II, p. 280, 16-17). Proclus parle également de deux Sirènes (voir plus loin). Contre ces interprétations antiques, M. Sulzberger a montré, exemples à l'appui, que l'usage du duel en grec homérique n'implique pas obligatoirement

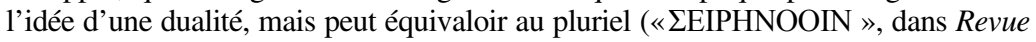
Belge de Philologie et d'Histoire 5, 1926, p. 939-942). Aussi ne faut-il pas supposer, comme le fait Walter Burkert (Lore and Science in Ancient Pythagoreanism, Cambridge, Harvard University Press, 1972, p. 364, note 74) sans pouvoir la préciser, une signification au fait qu'Homère parle de deux Sirènes.

4. Odyssée XII, 44-45.

5. Odyssée XII, 188.

6. Odyssée XII, 191.

7. Odyssée XII, 158.

8. Odyssée XII, 187.

9. Sur le caractère prophétique de l'abeille, le rapprochement entre abeille et prophétesse, et le symbolisme qui relie abeille et poète, voir Gabriel Germain, Genèse 
Peu de renseignements donc chez Homère. Par la suite, on leur attribua une généalogie ${ }^{10}$ : elles seraient filles du dieu-fleuve Achéloos et de la muse Terpsichore, ou du même Achéloos et de Stéropé, deux traditions qui se trouvent chez le pseudo-Apollodore ${ }^{11}$. Leurs noms et leur nombre varient suivant les traditions ${ }^{12}$.

Quoi qu'il en soit, du récit peu détaillé d'Homère, et dans une vision quelque peu réductrice ${ }^{13}$, on a tendance à retenir d'abord la composante mortifère que l'on peut tirer à la fois de la conséquence tragique de leur chant sur les marins, mais aussi du lieu où le poète les situe, à savoir une prairie aux bords blanchis par les ossements. En effet, la prairie dans laquelle résident les sirènes homériques est certes un endroit captivant et empreint de sensualité - c'est une prairie fleurie, dit le poète ${ }^{14}-$, à l'instar de la luxuriante prairie dans laquelle s'ébattent Coré et ses compagnes et où Terre fit pousser le narcisse, source de thambos, de sebas, qui piégea la jeune fille ${ }^{15}$.

de l'Odyssée. Le fantastique et le sacré, Paris, PUF, 1954, p. 387-390, qui rappelle notamment que le nom de $\mu \varepsilon ́ \lambda \_\sigma \sigma \alpha$ a été donné à certaines prêtresses et même à la Pythie; Liliane Bodson, IEPA ZRIA, Contribution à l'étude de la place de l'animal dans la religion grecque antique, Bruxelles, Palais des Académies (Académie Royale de Belgique. Mémoires de la Classe des Lettres, 63, fasc. 2), 1978, p. 37, souligne notamment comme caractère commun à l'abeille, à la prophétesse et à l'oracle de Delphes, leur lien avec les puissances chthoniennes; Françoise Bader, «Les Sirènes et la poésie», dans Danièle Conso, Nicole Fick et Bruno Poulle (éd.), Mélanges Kerlouégan, Paris, Les Belles Lettres, 1994, p. 17-42, qui, parmi les différentes étymologies du nom des Sirènes, fonde la sienne sur un rapprochement entre

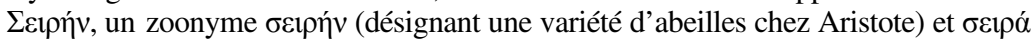
(«corde»): le rapprochement avec l'abeille s'explique par la composante poétique, le rapprochement avec la corde évoque l'aptitude à lier propre aux Sirènes, aptitude à mettre en rapport avec leur caractère de poétesses dans l'idée d'un «liage» poétique.

10. Apollonios de Rhodes, Argonautiques 4, 892.

11. Pseudo-Apollodore, Bibliothèque I, 3, 4 et I, 7, 10.

12. Voir notamment une scholie à Homère qui relève l'usage du duel chez le poète, mais note également d'autres traditions selon lesquelles les Sirènes seraient au nombre de quatre (Scholia Graeca In Homeri Odysseam II, p. 531, 1. 13-17 Dindorf) ou encore le scholiaste d'Hésiode qui signale qu'elles sont trois (Schol. Ap. Rhod. $\Delta$ 892, p. 298.7 Wendel = Hésiode fr. 27 Merkelbach et West).

13. Critiquée également par G. K. Gresseth, «The Homeric Sirens » [n. 2], p. 203 et note 4, et par B. Pauline Nugent, «The Sounds of Sirens; Odyssey 12.184-91 », dans College Literature 35, 2008, p. 45-54.

14. Odyssée XII, 159. L'île des Sirènes est également appelée Anthemoessa («fleurie») par Hésiode fr. 27 Merkelbach et West (référence en note 12).

15. Hymne à Déméter $1-19$ et particulièrement les vers 10 à 15 . À ce sujet, voir André Motte, Prairies et jardins de la Grèce antique. De la religion à la philosophie, Bruxelles, Palais des Académies (Académie Royale de Belgique. Mémoires de la Classe des Lettres, 61, fasc. 5), 1973, p. 35. 
Mais, si les Sirènes de l'Odyssée fascinent et séduisent, elles sèment également l'effroi et sont liées à la mort, comme l'est également, remarquons-le, la prairie où est enlevée Coré lorsqu'elle s'ouvre

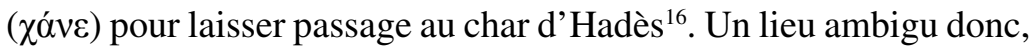
pour des créatures qui le sont tout autant, mais un lieu immédiatement associé aux Enfers car la prairie d'où les Sirènes font résonner leur chant est évocatrice d'une autre prairie qui se situe dans l'au-delà: celle où, selon Platon dans le mythe des Enfers du Gorgias, les trois juges, Minos, Éaque et Rhadamante, rendent leur sentence ${ }^{17}$. Dans ce mythe dont Socrate entame le récit en se référant à Homère, le lieu pourrait plutôt évoquer la prairie d'asphodèles de l'Odyssée $e^{18}$, «où habitent les âmes, images des défunts», que celle des Sirènes. Cependant, le mythe d'Er de la République, sur lequel nous aurons à revenir, évoque également un endroit «démonique», identifié à une prairie et situé à un croisement de quatre routes, ou, plus exactement, de deux fois deux routes qui se font face: deux routes montent au ciel et en descendent respectivement; deux autres descendent aux Enfers et en remontent respectivement. C'est là que se tiennent les juges qui dirigent les âmes soit sur la route montant au ciel, soit sur la route descendant aux Enfers ${ }^{19}$. Or, si l'on en revient à Homère, les conseils que donne Circé à Ulysse attestent que les Sirènes se trouvent au croisement de deux chemins: "Quand tes rameurs auront dépassé les Sirènes, deux routes s'offriront à toi » ${ }^{20}$. On notera encore que, d'une certaine manière, ces deux routes se dédoublent lorsqu'elles passent l'une entre les Roches Errantes, l'autre entre Charybde et Scylla ${ }^{21}$. Ce rapide rapprochement entre texte mythique et texte philosophique, qui se trouvera renforcé par une lecture plus attentive du mythe d'Er et de son interprétation néoplatonicienne,

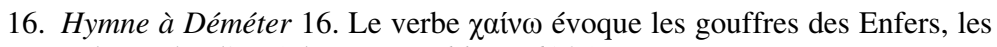
$\chi \alpha ́ \sigma \mu \alpha \tau \alpha$ du mythe d'Er (Platon, République 614c).

17. Platon, Gorgias 524a.

18. Odyssée XXIV, 13-14.

19. Platon, République 614a-e. Voir le schéma proposé par Jean-François Mattéi, Platon et le miroir du mythe. De l'âge d'or à l'Atlantide, Paris, PUF (Thémis Philosophie), 1996, p. 153.

20. Odyssée XII, 55-57.

21. Le jeu des particules de liaison, dans l'ensemble du passage (XII, 59-110), indique clairement ce dédoublement. En effet, les deux routes sont évoquées à partir du vers 59 pour la première ( $\varepsilon^{\prime} v \varepsilon v \mu \varepsilon \dot{v}$ ) et du vers 73 pour la seconde (oi $\delta \varepsilon \dot{)}$ ). Le $\tilde{\varepsilon} v \theta \varepsilon v \mu \varepsilon \dot{v} v$ est ensuite explicité par $\tau \tilde{n} \mu \varepsilon \dot{\varepsilon} v$ (vers 61) et $\tau \tilde{n} \delta \dot{\varepsilon}$ (vers 66), quant au

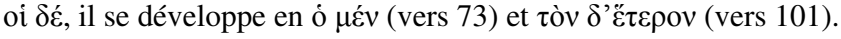


autorise ainsi à cerner les Sirènes comme des entités chthoniennes, compagnes de Perséphone, approche sans doute peu explicite dans le poème épique et suggérée surtout par le lieu qu'elles occupent, mais qui l'est de façon plus évidente chez Euripide par exemple:

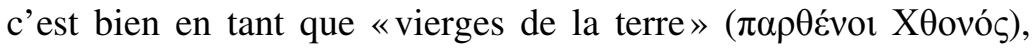
qu'Hélène les invoque, ainsi que Perséphone, les priant de faire écho à ses lamentations ${ }^{22}$. D'autres mythes encore, qui leur seront attachés plus tardivement, confirment ce rapport privilégié qu'elles entretiennent avec la reine des Enfers: Ovide rappelle qu'elles se trouvaient au nombre des compagnes de Proserpine dans la prairie où elle fut enlevée et qu'elles souhaitèrent des ailes pour partir à sa recherche par delà les flots ${ }^{23}$. Ce lien avec la mort et l'au-delà se voit confirmé par leur représentation sur des tombes, usage qui remonte au $v^{e}$ siècle avant notre ère ${ }^{24}$ et dans lequel elles apparaissent comme des figures secourables au pouvoir apotropaïque. Malgré ce rapport étroit entre le poème homérique, les traditions postérieures et la présence des Sirènes qui ornaient les tombeaux, on a eu tendance à distinguer nettement les Sirènes homériques, séductrices malfaisantes, et les Sirènes, «génies » protecteurs des tombeaux ${ }^{25}$. Nous voudrions pourtant souligner qu'êtres intermédiaires, placés à la croisée des chemins, figures dont l'ambiguïté se trouve déjà en germe chez Homère, les unes et les autres ont entretenu entre elles des rapports fondamentaux qui n'échappèrent pas non plus aux philosophes. Trois textes de Platon vont maintenant retenir notre attention.

22. Euripide, Hélène 167-178.

23. Ovide, Métamorphoses V, 554-560.

24. Voir Jacqueline Leclercq-Marx, La sirène dans la pensée et dans l'art de l'Antiquité et du Moyen Âge. Du mythe païen au symbole chrétien, Bruxelles, Académie Royale de Belgique, Classe des Beaux-Arts, 1997, p. 19.

25. Tel est l'avis que défend Paolo Rossi ( «Sirènes antiques. Poésie, philosophie, iconographie», dans Bulletin de l'Association Guillaume Budé 39, 4, 1970, p. 463481), avis qu'a fortement nuancé Ana Iriarte («Le chant-miroir des Sirènes », dans Métis 8, 1993, p. 147-159) qui démontre avec pertinence que «la Sirène, en tant que génie funèbre, participe de l'adresse intellectuelle propre aux vierges-oiseaux chanteuses qui abordent Ulysse» (p. 149). 


\section{Les SiRÈnes PlatonicienNes : ENTRE CIEL ET TERRE}

\section{Platon, Cratyle 403a-404b}

Dans le Cratyle, dans la discussion sur le nom d'Hadès, Socrate attribue aux hommes d'avoir commis beaucoup d'erreurs dans la compréhension de ce nom par crainte de la mort ${ }^{26}$. Il met le nom

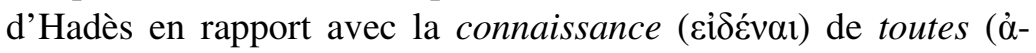
collectif ou intensif) les belles choses ${ }^{27}$. Hadès est omniscient et, aux dires de Socrate, un sophiste accompli, un philosophe qui retient les hommes (lorsqu'ils sont détachés du corps) par le désir de la vertu. Ses discours sont si beaux que les Sirènes elles-mêmes ne veulent pas quitter l'au-delà pour revenir ici-bas : elles y restent enchaînées, sous le charme ${ }^{28}$. C'est comme si Platon attribuait à Hadès la qualité d'être omniscient dont le poète avait doté les Sirènes, ainsi que le charme de la parole ; c'est comme si, en Hadès, elles avaient trouvé leur maître. Une chose est claire toutefois : leur localisation dans le monde infernal. Qu'est-ce qui peut justifier que Platon les ait, sans équivoque, placées là? Est-ce cette relation avec les Enfers qu'on ne peut manquer de relever à la lecture du poème homérique ? Ou sa connaissance d'une tradition selon laquelle, vaincues par Ulysse, elles se jetèrent dans la mer et périrent ${ }^{29}$ ? Cette question restera sans réponse; en revanche, elle nous mène à l'examen des autres extraits platoniciens dans lesquels cette localisation apparaît de façon moins tranchée.

\section{Platon, Phèdre 230b-c}

Enchantées par Hadès, les Sirènes n'en restent pas moins ellesmêmes enchanteresses. Et en tant que telles, elles sont apparentées aux cigales et proches des Muses. On se souvient en effet de l'enchantement qui, au début du Phèdre, saisit Socrate lorsqu'il accompagne Phèdre le long de l'Ilissos: la proximité d'une source consacrée aux Muses et à Achéloos (père des Sirènes, comme on l'a

26. Platon, Cratyle 403a.

27. Id., Ibid. 404b.

28. Id., Ibid. 403d-e.

29. La tradition du suicide des Sirènes, présente sur un célèbre vase attique à figures rouges du $\mathrm{v}^{\mathrm{e}}$ siècle AC (LIMC, VI, 2, pl. 632, $\left.\mathrm{n}^{\circ} 155\right)$, est rapportée plus tardivement par Lycophron, Alexandra 712-716. 
dit plus haut), une herbe tendre couvrant un pré en pente douce et le chant des cigale ${ }^{30}$ lui suggèrent bientôt un rapprochement entre les Sirènes et les cigales. Celles-ci, à l'heure de midi, font entendre leur chant, ce qui peut avoir deux effets: soit les hommes, sous le charme de leur voix, tombent dans le sommeil et leur esprit dans la paresse, soit ils continuent de converser et passent devant elles «comme devant des Sirènes, sans subir leurs enchantements». Dans ce cas, elles se réjouissent car, étant anciennement des hommes qui, sous le charme du chant des Muses, en oublièrent le boire et le manger, les cigales sont chargées d'aller rapporter aux Muses les hommages que leur rendent les hommes et, plus précisément, «à l'aînée Calliope et à sa cadette Uranie, elles annoncent ceux qui passent leur vie à philosopher et qui honorent leur art, l'art des Muses: entre toutes les Muses, ce sont elles qui, s'occupant du ciel et des discours divins et humains, font entendre la plus belle voix $»^{31}$. Cet extrait de Platon rapporte implicitement le nom des Sirènes à une des étymologies du

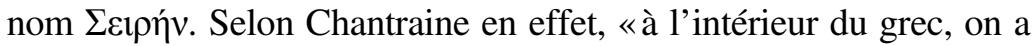

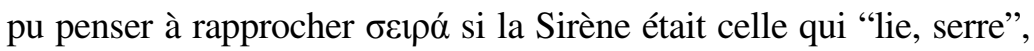
ou $\Sigma$ cípıot, ce qui évoquerait la grande chaleur de midi $»^{32}$. La chaleur de midi, l'enchantement du lieu, une prairie en pente douce, le charme du chant des cigales qui endormirait le commun des mortels, tout contribue à faire du lieu, aux dires mêmes de Socrate, un lieu divin $^{33}$, et, par la comparaison avec le chant des Sirènes, suggère que Platon devait avoir en mémoire le passage odysséen. Toutefois, les Sirènes ne sont peut-être pas convoquées ici qu'à titre de simple comparaison. J. Assaël a souligné le rôle qu'elles pourraient jouer dans ce contexte à la connotation mystérique évidente, en tant que divinités musiciennes amenant à une rencontre initiatique avec les Muses $^{34}$. Toutefois il ne nous semble pas que, si l'on doit les considérer comme hiérarchiquement inférieures à ces dernières, ce soit l'aspect sombre et chthonien des Sirènes, opposé au rayonnement olympien

30. Platon, Phèdre 230b-c.

31. Platon, Phèdre 259a-d.

32. Pierre Chantraine, Dictionnaire étymologique de la langue grecque, p. 993-

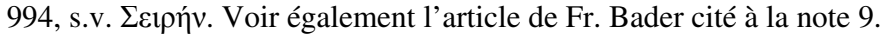

33. Platon, Phèdre 238c-d. Sur le rôle «actif» joué par ce lieu au fil du dialogue, voir A. Motte, Prairies et jardins [n. 15], p. 32-34.

34. Voir Jacqueline Assaël, «Sirènes, cigales et Muses. Degrés de l'initiation poétique dans les représentations mystériques des Grecs », dans Revue de l'Histoire des Religions 220, 2003, p. 131-151. 
des Muses qui autorise à le faire ${ }^{35}$. Au contraire, cette proximité des Muses, et plus précisément de Calliope, Muse de la philosophie, et d'Uranie, Muse de l'astronomie, ainsi que le rapport qui, par elles, s'établit entre la musique et l'ordre cosmique, ont estompé le caractère chthonien des Sirènes homériques et leur lien avec l'audelà que Platon a retenus dans le Cratyle. L'évocation des Sirènes dans le mythe des cigales, dont E. Moutsopoulos n'hésite pas à dire qu'il résume toute la doctrine platonicienne sur la musique, notamment dans les relations de celle-ci avec la science de l'art cosmique $^{36}$, semble bien les avoir élevées dans la sphère céleste. Pour autant, Platon reste ici fidèle à Homère car - on ne le souligne peut-être pas assez - c'est avant tout par leur chant que les Sirènes odysséennes séduisent. B. P. Nugent note le fait mais va plus loin: relevant chez Homère et d'autres poètes l'affirmation du caractère divin de la musique, de son pouvoir et son origine, elle défend l'idée que le rôle de la musique réside dans l'élévation de l'âme et qu'à cet égard, les Sirènes, par leur chant, servent d'intermédiaires ${ }^{37}$. Si nous ne sommes pas totalement convaincue par cette interprétation du texte d'Homère qui associe l'épisode des Sirènes à l'espoir d'une délivrance des liens de la condition humaine, il nous semble pertinent de relever ce rôle et cette place d'intermédiaires ${ }^{38}$. En effet, le double lien avec l'au-delà et avec le ciel, relevé respectivement dans le Cratyle et le Phèdre, loin de renfermer une contradiction, pourrait bien être l'expression platonicienne de l'ambiguïté essentielle dont sont marquées les Sirènes homériques et pourrait rendre compte, chez Platon, de la vision du poète «d'un au-delà à deux faces; l'une d'elles s'étend au jour, sur un plan horizontal, l'autre gît dans les profondeurs ténébreuses du sol», le leimôn étant le lieu où les deux faces communiquent entre elles ${ }^{39}$.

35. Comme le pense J. Assaël, «Sirènes, cigales et Muses» [n. 34], p. 132.

36. Evanghelos Moutsopoulos, La musique dans l' 'euvre de Platon, Paris, PUF, $1989^{2}$, p. 8.

37. B. P. Nugent, "The Sounds of Sirens» [n. 13], p. 45-54. Sur l'âme prisonnière du corps et les effets de la musique sur l'âme dans l'exégèse des poèmes homériques, voir déjà Félix Buffière, Les mythes d'Homère et la pensée grecque, Paris, Les Belles Lettres, 1956, p. 460-472.

38. Déjà relevés, avec d'autres modalités, par A. Iriarte ( «Le chant-miroir des Sirènes» [n. 25], p. 147-148) qui envisage les Sirènes comme intermédiaires entre les morts et les vivants.

39. A. Motte, Prairies et jardins [n. 15], p. 252, qui renvoie à l'Odyssée II, 489491 et X, 505-540. 


\section{Platon, République 616b-617d}

La lecture du mythe d'Er résout plus évidemment encore cette ambiguïté. Comme chacun sait, ce grand mythe eschatologique raconte comment Er, mort dans une bataille, revient à la vie douze jours plus tard et rapporte ce qu'il a vu et entendu dans l'au-delà, choisi par les dieux pour être leur messager. Son récit concerne le jugement des âmes, les récompenses et les punitions, mais également la structure de l'univers et plus précisément le mécanisme des mouvements célestes, avant le choix des genres de vie. Aussi est-ce dans le cosmos tout entier que Platon nous emmène. En effet, d'après le récit $\mathrm{d}^{\prime} \mathrm{Er}^{40}$, quand les âmes ont passé sept jours dans la prairie, croisement des routes du ciel et des enfers, elles partent pour un voyage de quatre jours. Au cinquième jour, elles arrivent à une lumière, comme une colonne ou un arc-en-ciel qui, d'en haut, enchaîne le ciel et la terre à la manière des cordes qui enserrent les bateaux. Cette lumière est comme un lien aux extrémités duquel est suspendu le fuseau de la Nécessité (Anankè) qui fait tourner toutes les sphères. Ce fuseau est, comme il se doit, constitué d'une tige, d'un crochet et d'un peson, c'est-à-dire d'un poids qui le fait tourner. La particularité du fuseau d'Anankè est qu'il s'agit d'un grand peson évidé avec des petits pesons à l'intérieur, au total huit pesons insérés les uns dans les autres, laissant voir en haut leurs bords comme des cercles autour de la tige. Le peson extérieur, le plus large et constellé, tourne sur les genoux de la Nécessité d'un mouvement uniforme: ce cercle extérieur est celui du cosmos; les pesons intérieurs tournent plus lentement et dans un sens contraire: ces sept cercles intérieurs sont les cinq planètes (Saturne, Jupiter, Mars, Mercure, Vénus), le Soleil et la Lune. Sur le haut de chaque cercle, une Sirène fait entendre une note unique et l'ensemble fait harmonie. Assises autour d'Anankè, les Moires - Lachésis, Clôthô et Atropos - chantent et font tourner les cercles: Clôthô avec la main droite le cercle extérieur, Atropos avec la main gauche les cercles intérieurs, Lachésis alternativement les uns et les autres de l'une ou l'autre main. Apparemment sans transition, le récit d'Er continue: les âmes se présentent à Lachésis pour le choix des 
genres de vie. Quand elles ont choisi leur vie, elles reçoivent de Lachésis un daimôn qui leur servira de gardien dans la vie. Guidée par ce démon, chaque âme est conduite vers Clôthô, et placée sous le fuseau de celle-ci (c'est la ratification du sort choisi), puis vers Atropos pour rendre irrévocable ce qui a été filé par Clôthô, et enfin au pied de la Nécessité, avant de se diriger dans la plaine du Léthé. Dans ce récit, Platon a repris et réinterprété des figures mythiques, les Moires et les Sirènes. Ces dernières sont au nombre de huit qui correspond au cosmos et aux sept planètes, et leur chant, une note chacune, c'est-à-dire l'accord de l'octave, constitue un ensemble harmonieux: c'est la fameuse «musique des sphères ${ }^{41}$; vues sous cet angle, elles sont manifestement plus célestes qu'infernales ou chthoniennes. Ici encore, Platon aurait-il conçu deux types de sirènes? La question surgit inévitablement dans notre mode de rationalité. Mais avant tout, ce serait oublier que cet «excursus astronomique» est inséré dans un mythe eschatologique où le mouvement des sphères est mis en rapport avec la destinée des âmes car les Moires, qui scellent le destin des âmes, font également tourner les cercles. Aussi sommes-nous tentée de penser que, dans le mythe d'Er, Platon a rendu compte de l'ambiguïté des Sirènes homériques.

\section{L'interprétation de Proclus}

\section{La catégorisation des Sirènes platoniciennes}

Dans l'Antiquité déjà, la cohérence de l'ensemble du mythe d'Er semblait ne pas aller de soi puisqu'on lit chez Proclus, rappelant que le but immédiat du mythe, en rapport avec celui de la République, est la justice et le jugement des âmes ${ }^{42}$ : «Mais pour ce but, un très petit nombre aurait amplement suffi de ce que Socrate développe dans le mythe sur les parts assignées par le sort dans l'Hadès, lorsqu'il traite de la bouche mugissante, des aventures tragiques d'Ardiée, des bénéfices de ceux qui ont saintement vécu. Mais que veulent nous dire, à la fois non mêlées au mythe et s'y

41. Sur la pertinence de cette appellation, voir W. Burkert, Lore and Science [n. 3], p. 351 et note 1.

42. Proclus, In rem publicam II, p. 97, 10-18 Kroll. 
mêlant, les révolutions des corps célestes, la royauté d'Ananké, les trois Moires, les lots, les vies, et les différences de toutes sortes dans les descentes des âmes en des êtres sans parole, en des êtres dotés de parole? Il faut en effet que toutes ces parties assurent une seule harmonie dans le discours mythique, ou que Socrate les ait cousues bout à bout sans raison, chose pour ainsi dire la plus absurde de toutes ${ }^{43}$. L'intention de Proclus est évidente: il entend clarifier le texte de Platon et en montrer la cohérence. On verra que, comme souvent, il en profite pour souligner l'accord entre Platon et Homère ${ }^{44}$.

Le commentaire du mythe d'Er s'ouvre par une dédicace à Marinus. Proclus annonce ensuite qu'il va passer en revue toutes les interprétations de ce mythe, particulièrement celle de Porphyre «que je déclare », dit-il, «avoir été plus que tous parfait exégète des vérités cachées dans le mythe ${ }^{45}$, et il répond précisément à la question de cette «digression» sur les révolutions célestes que nous venons d'évoquer. Selon lui, puisque le but immédiat de la République est la justice, Platon veut enseigner la république cosmique ou céleste, et donc montrer l'ordonnance des corps célestes et de tout ce qui se passe dans le ciel: «toutes les données de ce mythe-ci font voir clairement, je pense, le modèle dans le cosmos de la république socratique (...), elles montrent que le modèle de toute la république préexiste dans le tout $\gg^{46}$.

Dans cette république cosmique, il établit une hiérarchie dans laquelle les figures mythiques d'Ananké, des Moires et des Sirènes s'insèrent dans la hiérarchie dieux - démons - âmes, eux-mêmes modèles, dans la république cosmique, des gardiens - auxiliaires thètes de la république terrestre. Cette structure peut aboutir au schéma suivant:

43. Id., Ibid. II, p. 97, 18 - 98, 1 Kroll. Le passage platonicien ne laissa pas d'intriguer les modernes également. Ainsi Albert Rivaud parlait-t-il déjà d'une digression qui a quelque chose de singulier dans un récit relatif à la destinée de l'âme après la mort («Études platoniciennes I. Le système astronomique de Platon", dans Revue d'Histoire de la Philosophie 2, 1928, p. 8).

44. À ce sujet, voir notre article «Homère erre-t-il loin de la science théologique? De la réhabilitation du "divin" poète par Proclus», dans Kernos 15, 2002, p. 199210.

45. Proclus, In rem publicam II, p. 96, 13-15 Kroll.

46. Id., Ibid. II, p. 99, 10-17 Kroll. 


\begin{tabular}{|l|l|l|}
\hline $\begin{array}{c}\text { République cosmique } \\
\text { (modèle) }\end{array}$ & $\begin{array}{c}\text { République terrestre } \\
\text { (socratique) }\end{array}$ & Figures mythiques \\
\hline $\begin{array}{l}\text { 1. monade et triade } \\
\text { (causes hypercosmiques) }\end{array}$ & Ananké et Moires \\
$\begin{array}{l}\text { 2. Sirènes } \\
\text { 3. dieux célestes } \\
\text { 4. anges/démons, chefs } \\
\text { préposés aux } \underline{\text { âmes }}\end{array}$ & gardiens \\
5. juges & $\underline{\text { auxiliaires }}$ & Sirènes \\
6. exécuteurs & & \\
\hline
\end{tabular}

Dans cette structure, où le rôle des Sirènes est de maintenir l'harmonie du Tout par la musique, le fuseau est le monde. Selon Proclus qui prétend dévoiler une «façon de voir plus vraie et plus conforme au dernier degré de l'initiation », le fuseau d'Anankè est le nom mythique que Platon a donné au ciel car le ciel appartient aux Moires et que c'est par lui qu'elles tressent et filent toute la destinée pour les êtres qui viennent à exister ${ }^{47}$, et les Sirènes font partie des «hypostases divines et incorporelles qui président au mouvement du fuseau $»^{48}$. Proclus leur attribue l'essence et le rang d'âmes douées de vie intellective car, dit-il, elles sont antérieures aux corps et douées d'un mouvement circulaire. Selon lui, ces âmes sont nommées «Sirènes » par Platon qui a voulu montrer en cela que l'accord qu'elles mettent dans les cercles est de nature corporelle. Et il précise même que Platon les a appelées Sirènes «célestes », ce qui relève de la surinterprétation car le mot oủ $\rho \alpha v i ́ a \varsigma$ ne figure pas dans le texte de Platon. La raison pour laquelle Platon appellerait ces Sirènes «célestes» serait de les distinguer des Sirènes liées à la génésis. Celles-ci, selon Proclus, sont celles du Phèdre 259a (voir ci-dessus) et, par delà, celles de l'Odyssée puisqu'il faut les dépasser dans la navigation comme Ulysse. Proclus, se fondant sur l'usage homérique du duel ${ }^{49}$, les compte au nombre de deux: elles constituent une dyade ${ }^{50}$ et se distinguent par là des Sirènes dites «célestes» ou «ouraniennes» qui sont au nombre de huit, une par cercle, la première, sur le cercle extérieur, étant une monade à la

47. Id., Ibid. II, p. 203, 30 - 204, 22 Kroll.

48. Id., Ibid. II, p. 236, 22-23 Kroll.

49. Voir ci-dessus (note 3 ) ce qu'il faut en penser.

50. Proclus, In rem publicam II, p. 238, 23-28 Kroll. 
tête de l'hebdomade. Aussi, poursuit Proclus, si la monade est à la tête de sept, la dyade, constituée par les Sirènes de l'Odyssée, ne peut être qu'à la tête de $2 \times 7$ : selon lui, cela implique l'existence de Sirènes infernales qui, si on comprend bien, doivent être au nombre de quatorze. Ces dernières sont celles du Cratyle que Proclus évoque partant de son commentaire au mythe d'Er et faisant ainsi le lien entre les trois textes où Platon évoque les Sirènes, à savoir le Phèdre, le Cratyle et la République. Proclus ne fait d'ailleurs ici que réaffirmer l'existence de Sirènes chthoniennes, ce qu'il avait déjà dit précédemment et de la même façon, à savoir en confirmant un détail du mythe d'Er par une citation du Cratyle. En effet, commentant plus précisément cette phrase de Platon: «Tels étaient à peu près les peines et les châtiments, ainsi que les bienfaits correspondants $»^{51}$, phrase qui succède à la description par Platon des punitions que subissent les criminels, il entreprend de répondre à la question de savoir ce que sont les «bienfaits» correspondants aux châtiments ? ?h $^{52}$ Chez Platon, on comprend qu'il s'agit de la joie d'effectuer la remontée dans le silence, c'est-à-dire sans entendre le bruit ( $\tau$ ò $\varphi \theta \dot{\varepsilon} \gamma \mu \alpha$ ) que fait l'ouverture (la sortie) quand un mauvais veut sortir, signal auquel des hommes de feu s'emparent de lui, lui font subir des maux atroces et l'emmènent dans le Tartare. Voici l'interprétation de Proclus: ces bienfaits accordés aux justes sont d'être en compagnie des démons Ploutoniens et, au bout d'un cheminement fait d'apparitions et de purifications, de communiquer avec Plouton lui-même et Korè, comme dans les mystères d'Éleusis. Proclus confirme cette surprenante interprétation par cette citation du Cratyle: "personne ne veut revenir de l'autre monde dans celui-ci, pas même les Sirènes, un charme les tient liées $»^{53}$, citation qui l'autorise à avancer l'existence de Sirènes infernales.

Par conséquent, le commentaire de ce passage du mythe d'Er permet à Proclus de souligner la cohérence interne de l'œuvre même de Platon, et, en l'occurrence, de rationaliser, de "catégoriser» les Sirènes platoniciennes. On peut douter que Platon ait tenu à distinguer trois types de Sirènes; il avait dû s'accommoder du mystère lié aux Sirènes de l'Odyssée et au lieu qu'elles occupent, la

51. Platon, République 616a-b.

52. Proclus, In rem publicam II, p. 184, 29 - 185, 19 Kroll.

53. Platon, Cratyle 403d. Voir plus haut et note 26. 
prairie, dont l'image, pour reprendre les termes d'André Motte, est celle «d'une plénitude vitale que traverse le cycle de la naissance et de la mort $\gg^{54}$. Proclus parvient également à mettre en accord Homère et Platon, et resserre encore l'adéquation entre le texte philosophique et le texte mythique en attachant les trois types de Sirènes aux trois dieux à qui, selon Homère, échut le pouvoir sur les trois parties du monde ${ }^{55}$ : «selon Platon, il y a trois espèces de Sirènes : les célestes, dépendant de Zeus, les terrestres, dépendant de Poséidon, les souterraines, dépendant de Pluton $»^{56}$. Les premières, au nombre de huit, sont celles de la République, les deuxièmes, au nombre de deux, sont celles de l'Odyssée et du Phèdre, les troisièmes, au nombre de quatorze, sont celles du Cratyle.

Encore fallait-il que cette cohérence s'inscrive dans le système propre de Proclus. Aussi, dans la république cosmique, s'agissant des Sirènes «célestes» de la République et du rapport entre la monade/Ananké, la triade/Moires et les Sirènes, Proclus place-t-il les Moires à un rang inférieur à Anankè et supérieur aux Sirènes. Or, il qualifie cette triade d' "à la fois hypercosmique et encosmique $»^{57}$. Dans le système fortement hiérarchisé de Proclus, cela implique nécessairement que la monade est hypercosmique et les Sirènes encosmiques. Cette hiérarchie, qui était loin d'aller de soi dans le texte de Platon, correspond en effet à sa propre hiérarchisation du divin, exposée au livre VI de la Théologie platonicienne, où les dieux du monde se divisent en dieux hypercosmiques, en dieux hypercosmiques-encosmiques et en dieux encosmiques (célestes et sublunaires $)^{58}$. Ainsi, les révolutions célestes se produisent dans un mouvement qui procède depuis le haut jusqu'en bas de la manière suivante: Ananké est assise et ne touche pas le fuseau (monade hypercosmique), les Moires sont assises également mais touchent le fuseau et le meuvent de leurs mains sans être entraînées par le mouvement (triade hypercosmique-encosmique) et enfin les Sirènes, sur les cercles, accompagnent le mouvement (encosmiques) ${ }^{59}$.

54. A. Motte, Prairies et jardins [n. 15], p. 158-159.

55. Iliade XV, 185.

56. Proclus, In rem publicam II, p. 239, 7-9 Kroll.

57. Id., Ibid. II, p. 239, 26 Kroll.

58. Voir l'introduction à Proclus, Théologie platonicienne, livre I, texte établi et traduit par Henri-Dominique Saffrey et Leendert Gerrit Westerink, Paris, Les Belles Lettres $(C U F), 1968$, p. LXVI-LXVII.

59. Proclus, In rem publicam II, p. 240, 3-11 Kroll. 
Dans une perspective tout à fait néoplatonicienne, Ananké, Moires et Sirènes sont ainsi les maillons d'une chaîne qui se déploie depuis le haut jusqu'en bas, «jusqu'aux derniers des êtres», et qui est symbolisé ici par le fil de la quenouille ${ }^{60}$.

C'est ici que, pour renforcer encore son discours, Proclus fait intervenir le rituel théurgique, le mettant en adéquation avec le texte philosophique: «C'est pour cette raison que Platon a comparé les révolutions à un fuseau et à des pesons, car dans les cérémonies sacrées aussi, on a coutume de prendre comme symboles des Moires ces objets, quenouilles, fuseaux, fils $»^{61}$. On apprend dans la suite immédiate du texte que le rituel auquel Proclus fait allusion est le rituel de la télestique, à entendre ici dans le sens de l'évocation du dieu et l'illumination de sa statue: le dieu, évoqué par les symboles qui lui sont attachés, illumine et anime sa statue. Pour Proclus, le rituel vient confirmer que les Moires, en imprimant le mouvement aux sphères, donnent du même coup la vie: «Il ne faut pas penser que, quand elles ordonnent et meuvent toutes choses, elles meuvent sans donner le principe vital. Car ceux qui illuminent les statues d'ici-bas ne les illuminent pas sans principe vital $»^{62}$. Proclus doit encore expliquer que les Moires, figures mythiques établies par lui dans la hiérarchie du divin au rang hypercosmique-encosmique, étant incorporelles, puissent être vues et entendues par Er. Une fois encore, «tout ceci s'éclaire à la lumière de notre art hiératique » ${ }^{63}$, dit-il: prenant à témoin les Oracles chaldaïques qui disent que «à cause de vous des corps ont été attachés à nos autophanies ${ }^{64}$ et s'appuyant sur le concept de participation, il avance que c'est l'âme qui, participant à la fois du participé incorporel et du participant corporel, par la faculté imaginative, voit et entend les Moires avec les attributs que leur a donnés le mythe, à savoir les trônes, les bandelettes et les robes blanches. Celles-ci sont comme les robes symboliques dont sont parées les statues animées par la télestique ${ }^{65}$. Proclus réinterprète ainsi le texte de Platon et lui attribue un langage symbolique, compréhensible à la lumière de la théurgie; il y décrypte

60. Id., Ibid. II, p. 240, 25-27 Kroll.

61. Id., Ibid. II, p. 240, 22-25 Kroll.

62. Id., Ibid. II, p. 241, 2-5 Kroll.

63. Id., Ibid. II, p. 243, 16-17 Kroll.

64. Fr. 142 des Places.

65. Proclus, In rem publicam II, p. 246, 23-26 Kroll. 
un message caché qui est le propre des «mythoplastes $»^{66}$. En faisant de Platon un faiseur de mythes, il peut expliquer ce qu'il considère comme des inversions dans le discours platonicien et effectuer un redressement qui met en concordance son discours avec celui de son Maître. Ainsi explique-t-il, par exemple, la phrase de Platon qui dit que les Moires chantent des hymnes en harmonie avec les Sirènes

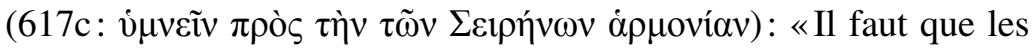
harmonies des Sirènes suivent les hymnes chantés par les Moires. Mais Platon, cachant la chose, a montré à l'inverse les Moires chanter des hymnes en harmonie avec les Sirènes $\gg^{67}$. Il est évident que le texte de Platon ne pouvait pas, dans le système hiérarchique qu'impose Proclus à celui-ci, supposer une antériorité, et donc une supériorité, des Sirènes par rapport aux Moires, qui aurait compromis la symphonie entre le discours de Platon et le sien propre.

\section{Les Sirènes, servantes des Muses et d'Apollon Musagète}

Proches des Sirènes sont également les Muses. Comme les Sirènes, les Muses apparaissent marquées d'une ambivalence qui tient de leur caractère à la fois chthonien et céleste. En effet, si elles apparaissent dans la mythologie au nombre des divinités célestes, elles n'en possèdent pas moins un aspect chthonien qui leur vient de leur affinité avec la nature: leur lieu d'inspiration est souvent une prairie (c'est dans les pâturages de l'Hélicon qu'Hésiode a reçu sa vocation de poète $\left.{ }^{68}\right)$. Comme les Sirènes de l'Odyssée qui savent tout, les Muses savent «ce qui est, ce qui fut, ce qui sera $»^{69}$. Cette proximité entre les Sirènes et les Muses, dévoilée par les textes mythiques, se rencontre également dans les textes philosophiques. Nous avons rappelé ci-dessus que, dans le Phèdre, Platon établit un rapprochement entre les Sirènes et les cigales, et que celles-ci sont antérieures aux Muses et à leur service. Le lien qu'établit Platon entre les Muses et les Sirènes a été perçu dès l'Antiquité au point que Plutarque attribue à Platon un rapprochement étymologique «étrange » entre le nom des Sirènes et le verbe elpeıv (dire), consistant

66. Id., Ibid. II, p. 238, 3 Kroll.

67. Id., Ibid. II p. 250, 16-19 Kroll.

68. Pour d'autres références, voir A. Motte, Prairies et jardins [n. 15], p. 29, note 11 et p. 301-302, note 65 .

69. Hésiode, Théogonie 38. 
à appeler les Muses «des Sirènes proclamant les choses divines et les

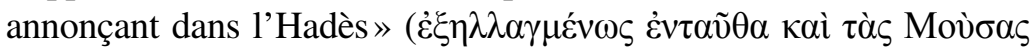

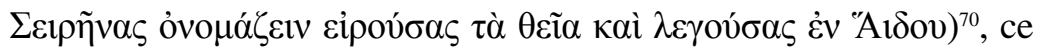
qui ferait des Sirènes une sorte de pendant infernal des Muses. Ce passage de Plutarque mérite qu'on s'y arrête un moment. Dans une discussion sur le nombre des Muses, l'intervention du Péripatéticien Ménéphylos fait glisser le propos sur les Sirènes et établit la relation

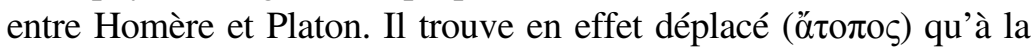
place des Muses Platon assigne les Sirènes aux révolutions éternelles et divines, alors qu'elles sont des divinités qui ne sont ni bienveillantes ni dévouées envers les hommes ${ }^{71}$, réflexion qui témoigne de la perception négative que devait d'ordinaire susciter l'évocation des Sirènes homériques. Le porte-parole de Plutarque, Ammonios, avance alors cette interprétation: «Les Sirènes d'Homère nous causent dans le mythe une peur qui n'a pas de raison d'être; au contraire, celui-là a dit à mots couverts à juste titre que la puissance de la musique n'est ni inhumaine ni funeste, mais que dans les âmes qui sont parties d'ici pour aller là-bas, à ce qu'il semble, et errent après la mort, elle fait naître l'amour des choses célestes et divines, et l'oubli des choses mortelles, elle les possède et les charme par le chant; et elles, sous l'effet de la joie, les suivent et accompagnent leurs révolutions. Et ici, comme un écho affaibli de cette musique, arrivant à nous, appelle nos âmes par des paroles et leur rappelle les réalités d'alors. Mais les oreilles de la plupart sont obstruées et bouchées par les obstacles des passions charnelles et non par des morceaux de cire, tandis que l'âme, par de bonnes dispositions naturelles, entend et se souvient, et la passion qu'elle éprouve n'est en rien inférieure aux amours les plus furieuses, elle souhaite ardemment se délivrer du corps et regrette de ne pouvoir y parvenir; cependant, je ne suis pas tout à fait d'accord avec cela: il me semble que, comme il appelle les axes du ciel «fuseaux» et «quenouilles», et «pesons » les astres, Platon, étrangement, appelle ici

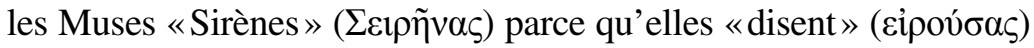
et proclament les choses divines dans l'Hadès ${ }^{72} »$. Si Ammonios dit ne pas adhérer tout à fait à cette interprétation, il n'en reste pas moins que ce passage atteste non seulement les spéculations dont

70. Plutarque, Propos de table IX, 14, 6 745F.

71. Id., Ibid. IX, 14, 5, 745C.

72. Id., Ibid. IX, 14, 6, 745D-F. 
ont fait l'objet les Sirènes homériques et les Sirènes du mythe d'Er dans les cénacles platoniciens, mais aussi la volonté de donner au texte poétique une interprétation qui fasse se rejoindre le poète et le philosophe dans une approche positive des Sirènes: ici, dans une perspective platonicienne, les Sirènes homériques, par leur musique, libèrent l'âme du corps et la sauvent, étant entendu que cela ne peut arriver qu'à l'âme du sage (Ulysse) et non à celle du commun des mortels (les rameurs aux oreilles bouchées, c'est-à-dire les hommes «assourdis» par les passions) ${ }^{73}$. À la présence des Sirènes dans le mythe d'Er, c'est-à-dire à l'étonnante absence des Muses, Ammonios préfère cependant trouver l'explication étymologique mentionnée ci-dessus. Proclus ira plus loin. On ne s'étonnera guère que, dans son programme de hiérarchisation du divin, Proclus ait tenu à assigner un rang aux Muses, un rang qui ne pouvait être que supérieur à celui des Sirènes, comme invite à le concevoir le mythe des cigales du Phèdre. Aussi, bien que les Muses soient absentes du mythe d'Er, Proclus affirme que «par le fait d'être entraînées dans la ronde des cercles, les Sirènes sont inférieures aux Muses $\gg^{74}$. Enfin, inférieures aux Muses mais proches d'elles, les Sirènes ne peuvent qu'être sous la houlette d'Apollon Musagète. Cette présence du dieu, Proclus a manifestement tenu à l'affirmer. Il convient de montrer comment et pourquoi.

En République 377c sq., Platon traite de l'éducation des gardiens qui comporte la musique pour les âmes et la gymnastique pour les corps. La musique comporte, selon lui, une partie qui concerne les paroles et les mythes ${ }^{75}$. Aussi traite-t-il la question des fables mensongères, dans lesquelles «on représente les dieux et les héros d'une manière erronée ${ }^{76}$. Il appartiendra aux fondateurs d'État de veiller à ce que les poètes représentent le dieu tel qu'il est et donc de savoir quels modèles ils devront suivre pour en parler ${ }^{77}$. Dans son commentaire, après avoir relevé toute une série de points de vue apparemment contradictoires chez Platon ( «Si l'art poétique

73. À ce sujet, voir F. Buffière, Les mythes d'Homère [n. 37], p. 476-481, qui souligne le pythagorisme de ce type d'exégèse.

74. Proclus, In rem publicam II, p. 237, 20-22 Kroll. La suite du texte est corrompue et ne permet pas d'en savoir plus.

75. Platon, République 398b.

76. Id., Ibid. $377 \mathrm{e}$.

77. Id., Ibid. 379a. 
a, selon Platon, quelque chose de divin, comment est-il banni de la divine république ? $\gg^{78}$ ), Proclus détermine dix questions à traiter concernant les opinions de Platon sur la poétique, la dixième étant: "Quel est le poète dans le tout, sur le modèle de qui le poète aussi d'ici-bas obtiendra sa propre fin? Car il n'est aucun des vrais biens qui n'existe dans le tout bien avant d'exister dans les parties. $\gg^{79}$ Reprenant l'affirmation de Platon selon laquelle le poète est un imitateur et qu'il doit, dans son imitation, produire un objet semblable à son modèle, Proclus prolonge donc la réflexion platonicienne par cette dernière question : quel est le poète du Tout? Il y répond en établissant la structure suivante dans le Tout: au sommet, le Politique et, en-dessous, des collaborateurs : le Stratège, l'Orateur public, le Médecin, et le Poète, tous ceux-ci sont les modèles des politiques, des stratèges, des orateurs, des médecins et des poètes d'ici-bas. Dans cette structure platonicienne, absente du texte de Platon, Proclus trouve une place pour les dieux: le Politique $=$ Zeus, le Stratège $=$ Arès, l'Orateur public $=$ Hermès, le Médecin = Asclépios. Le Poète est Apollon «qui produit des imitations douées d'harmonie et de rythme $»^{80}$. Or, qui dit harmonie dans le tout dit musique des sphères, donc chant des Sirènes célestes. Par conséquent, Proclus attribue à Apollon de présider aux révolutions des sphères: "Celui qui effectue avec lui [scil. Zeus] tout l'ordre dans le tout au moyen de mouvements vifs et lents et de révolutions plus courtes ou plus longues n'est pas un autre qu'Apollon ${ }^{81}$, et, faisant le lien avec les Sirènes du mythe d'Er, il dit immédiatement après qu' «Apollon meut les Sirènes à chanter en émettant un seul son, un seul ton, comme le dit le mythe au $\mathrm{X}^{\mathrm{e}}$ livre de la République ${ }^{82}$. Enfin, comme s'il n'était pas suffisant de souligner la cohérence interne de la République, il renvoie encore au texte du Timée sur le mouvement du Même et de l'Autre, assignant à Apollon (absent du Timée) «de mouvoir les cercles des Âmes divines qui tournent avec mesure selon des mouvements bien rythmés ${ }^{83}$. Il est vrai que la référence au Timée

78. Proclus, In rem publicam I, p. 42, 8-9 Kroll.

79. Id., Ibid. I, p. 43, 22-25 Kroll.

80. Id., Ibid. I, p. 68, 29-69, 1 Kroll.

81. Id., Ibid. I, p. 68, 26-29 Kroll.

82. Id., Ibid. I, p. 69, 10-12 Kroll.

83. Id., Ibid. I, p. 69, 12-14 Kroll. 
s'invite d'elle-même: les deux cercles formés par le démiurge lors de la construction de la voûte céleste, l'un, extérieur et ne comportant pas de division (le cercle du Même), l'autre, intérieur et divisé en six cercles (le cercle de l'Autre), rappellent les pesons du fuseau d'Ananké. Il n'en reste pas moins que Proclus répond à une question sur la poétique que Platon ne pose pas, disant que le poète d'ici-bas doit avoir son regard fixé sur Apollon, opinion qu'il étaye en allant puiser dans deux textes de Platon sur les sphères célestes, installant de surcroît les dieux dans cette construction. Dans une démarche identique qui, cette fois, renvoie du Timée à la République, il affirme encore dans le Commentaire sur le Timée: «Les Anciens ont donné la présidence sur le tout aux Muses et à Apollon Musagète, l'un procurant l'unité unique de l'harmonie totale, les autres maintenant ensemble la progression divisée de cette harmonie et ayant accordé leur nombre avec les huit Sirènes de la République » ${ }^{84}$. Celles-ci sont les Sirènes dites «célestes» par Proclus, inférieures aux Muses et à Apollon Musagète.

Le Zeus qui est ici détenteur du modèle de la politique est celui du Protagoras et nous avons pu montrer ailleurs qu'il a été identifié par Proclus au démiurge du Timée ${ }^{85}$. Le lien entre la République et le Timée n'en est que plus étroit.

Inférieures aux Muses sont également les Sirènes dites de la génésis, comme les appelle Proclus pour les distinguer des Sirènes célestes $^{86}$. En République 546a sq., Socrate dit se faire, comme Homère, le porte-parole des Muses sur la question de l'introduction de la discorde dans la cité qu'il explique par les périodes de fécondité et de stérilité des animaux, périodes que ne seront pas capables de discerner ceux qui guident l'Etat, tout excellents qu'ils sont. Ils engendreront des enfants quand il ne faut pas. Ces enfants deviendront des chefs qui négligeront la musique (c'està-dire les Muses) et privilégieront la gymnastique. Contrairement à la génération divine qui se fait dans une période correspondant à un nombre parfait qui n'est pas révélé par Platon, la génération des hommes se fait dans une période exprimée par un nombre qui

84. Id., In Timaeum II, p. 208, 9-14 Diehl.

85. Voir notre article «De l'omnipotence de Zeus : entre tradition mythologique et doctrines philosophiques et théologiques», dans Revue des Sciences Philosophiques et Théologiques 87, 2003, p. 248.

86. Voir ci-dessus. 
pourrait être obtenu à partir des nombres 3,4 et 5 du triangle nuptial, nombres qui, à la suite de diverses opérations, engendrent deux harmonies ${ }^{87}$. Ce sont ces harmonies qui commandent aux bonnes et aux mauvaises naissances. Dans son interprétation de ce passage, Proclus rapporte ces deux harmonies aux Muses et à certaines Sirènes: la première convient au divin et sauve les âmes, l'autre est liée à la génération et joint les âmes aux choses matérielles. Il renvoie ensuite à Homère car ces Sirènes de la génération sont celles de l'Odyssée : «celui qui gagne le large et se sauve lui-même passera à côté d'elles dans sa navigation, poursuivant l'harmonie meilleure et vraiment propre aux Muses, tandis que la masse se réjouira d'être enchaînée par les Sirènes et de demeurer dans la nature et ses délices $»^{88}$. Ainsi, l' accord entre Homère et Platon se trouve-t-il une fois de plus scellé. Sans doute cette mise en adéquation par Proclus du discours du poète et de celui du philosophe a-t-elle pu s'imposer d'elle-même car en fin de compte, et bien qu'il l'ait chassé de sa cité, chez le philosophe, le poète n'est jamais vraiment loin.

\section{Conclusion : du Chant des Sirènes à l'oracle de Delphes}

Enchanteresses, sensuelles et séductrices, mais mortifères, résidant dans une prairie, lieu évocateur dans l'imaginaire grec de ravissement, mais aussi sinistrement marqué par la mort, les Sirènes omniscientes auxquelles Ulysse n'échappa que par ruse et grâce à la fermeté de ses compagnons qui refusèrent de le détacher de son mât appartiennent à ces figures mythiques qui ont trouvé une place dans le discours des philosophes. Figures ambiguës, à la croisée

87. Sur ces opérations complexes et le mystère du nombre parfait de Platon, voir notamment Marc Denkinger, "L'énigme du nombre de Platon», dans Revue des Études Grecques 68, 1955, p. 38-76. Plus récemment, Godefroid de Callataÿ («Il numero geometrico», traduit en italien par Franco Ferrari dans Mario Vegetti (éd.), Platone, La Repubblica, traduzione e commento, vol. VI, Libri VIII-IX (Elenchos, XXVIII-6), Napoli, 2005, p. 169-187), rappelle l'interprétation mathématique et les calculs qui ont mené à déterminer la valeur de ce nombre «géométrique » et, parlant du «secret des muses » qui recouvre ce nombre, seul accessible à quelques initiés (ceux qui contrôleront les naissances), il fait remarquer que Platon n'a pas révélé à ses lecteurs le rapport entre ce nombre «géométrique» et les périodes de reproduction des hommes (voir plus particulièrement p. 183).

88. Proclus, In rem publicam II, p. 68, 10-14 Kroll. 
des chemins, elles pourraient même avoir servi à consolider le lien que certains d'entre eux ont voulu établir entre leurs théories cosmologiques et eschatologiques. Platon les fait présider aux sphères célestes, dont le chant des Sirènes est la musique, mais dont le mouvement détermine le sort des hommes, dans un au-delà à deux plans, l'un céleste, l'autre infernal, dont le point de jonction est la prairie. Conciliant le discours philosophique et le discours mythique, Proclus lève toute ambiguïté et, assurant la cohérence à la fois de l'exposé de Platon et du sien, avance trois catégories de Sirènes, les célestes, les terrestres et les souterraines, les secondes étant celles de l'Odyssée. Dans cette perspective, où le mythe est venu nourrir la réflexion philosophique, le rite n'est jamais loin non plus. Aussi terminerons-nous par cette énigmatique mention du chant des Sirènes qui le rattache à l'oracle de Delphes et qu'on peut lire dans la Vie de Pythagore de Jamblique. Parmi les maximes orales, appelées akousmata, qui doivent préparer les disciples de Pythagore à la sagesse, il en est qui sont rangées sous la rubrique «qu'est-ce que c'est?». Et parmi les exemples de maximes du type «qu'est-ce que c'est?», celle-ci: «Qu'est-ce que l'oracle de Delphes? - La tétraktys, c'est-à-dire l'harmonie où résident les Sirènes » ${ }^{89}$. Comme on le lit dans le Protreptique, ces maximes orales sont des symboles qui rendaient les entretiens et les écrits que s'échangeaient les membres de l'école pythagoricienne inaccessibles et inintelligibles

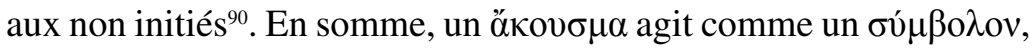
un signe de reconnaissance ${ }^{91}$ : pour les Pythagoriciens, l'oracle de Delphes est symboliquement la tétraktys, c'est-à-dire la décade qui, étant la somme de $1+2+3+4$, comporte des propriétés que l'on peut rapporter non seulement aux nombres et à la géométrie, mais aussi aux intervalles musicaux, et à la constitution du monde. Il

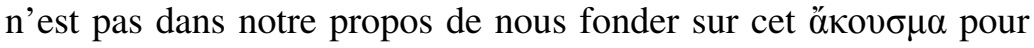
affirmer le pythagorisme de Platon. Nous y soulignerons plutôt la volonté de Jamblique de mettre en accord Pythagore, Homère et Platon: la tétraktys renvoie à Pythagore, les Sirènes renvoient à Homère et l'harmonie céleste à Platon. Qu'est-ce qui a bien pu permettre le lien entre la décade, qui, chez les Pythagoriciens,

89. Jamblique, Vie de Pythagore 82.

90. Jamblique, Protreptique 21.

91. Voir J.-Fr. Mattéi, Pythagore et les Pythagoriciens, Paris, PUF (Que sais-je ?), 1993, p. 28-31. 
fonde l'harmonie, et l'oracle de Delphes ${ }^{22}$, si ce n'est la présence d'Apollon? Apollon aux multiples figures, si bien évoquées par Platon dans les jeux étymologiques du Cratyle: il est notamment le dieu «sincère» $(\dot{\alpha} \pi \lambda \circ \tilde{o} \varsigma)$, c'est-à-dire le dieu de la divination,

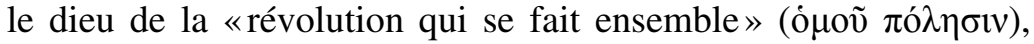
c'est-à-dire de l'harmonie, celle des sphères et celle de la musique ${ }^{93}$. Comment s'étonner dès lors que l'harmonie céleste ait été placée sous le patronage du dieu de Delphes? Et les Sirènes n'ont-elles pas elles-mêmes favorisé ce rapprochement? Par leur chant, elles sont les compagnes des Muses et d'Apollon Musagète. Chthoniennes et célestes, elles rappellent que ce n'est qu'après avoir tué le dragon femelle, symbole de l'ancien oracle de la Terre, que le dieu de la lumière a bâti son sanctuaire. Omniscientes et prophétiques, elles prennent naturellement place aux côtés du dieu de l'oracle. Inattendu destin décidément que celui des Sirènes odysséennes, étranges figures dont le mystère originel a permis, de Pythagore à Proclus, les plus étonnantes et les plus savantes constructions.

cvlieffe@ulb.ac.be

Université Libre de Bruxelles

Avenue F. D. Roosevelt 50, CP 175

1050 Bruxelles

Belgique

92. Ce lien établi par les Pythagoriciens entre l'oracle de Delphes et l'harmonie des Sirènes a déjà été étudié par Marie Delcourt, L'oracle de Delphes, Paris, Payot, 1981, p. 250-252.

93. Platon, Cratyle 405c-e. 